\title{
EXTREMAL AND MONOGENIC ADDITIVE SET FUNCTIONS
}

\author{
DETLEF PLACHKY
}

\begin{abstract}
The extreme points of the convex set of all additive set functions on a field, which coincide on a subfield are characterized by a simple approximation property. It is proved that a stronger approximation property is characteristic for a so-called monogenic additive set function on a field, which can be generated uniquely by an additive set function on a subfield. Finally it is shown that a simple decomposition property must hold if the convex set above has a finite number of extreme points.
\end{abstract}

1. Definitions. In the terminology of [4] let $b a\left(\Sigma, \nu, \Sigma^{\prime}\right)$, denote the set of all $\mu \in b a\left(S, \Sigma^{\prime}\right)$ with $\mu \geq 0$ and $\mu(S)=1$, such that $\mu \mid \Sigma=\nu$, where $\Sigma^{\prime}$ is a field of subsets of a set $S, \Sigma$ is a subfield of $\Sigma^{\prime}$ and $\nu \in b a(S, \Sigma)$ with $\nu \geq 0$ and $\nu(S)=1$. The set $c a\left(\Sigma, \nu, \Sigma^{\prime}\right)$, where $\Sigma$ and $\Sigma^{\prime}, \Sigma \subset \Sigma^{\prime}$, denote $\sigma$-fields and $\nu$ is a probability measure on $\Sigma$, is defined in the same way.

2. Main results. From the techniques of Douglas [3] it follows that $\mu \in c a\left(\Sigma, \nu, \Sigma^{\prime}\right)$ is an extreme point iff $L_{1}(S, \Sigma, \nu)$ is dense in the set $L_{1}\left(S, \Sigma^{\prime}, \mu\right)$ of all (equivalence classes of) $\mu$-integrable functions with respect to the norm topology. The completeness of $L_{1}(S, \Sigma, \nu)$ implies that $\mu \in c a\left(\Sigma, \nu, \Sigma^{\prime}\right)$ is an extreme point iff for all $A \in \Sigma^{\prime}$ there exists $B \in \Sigma$ with $\mu(A \triangle B)=0$. Stone's representation theorem yields the following obvious generalization to $b a\left(\Sigma, \nu, \Sigma^{\prime}\right)$ :

THEOREM 1. It holds that $\mu \in b a\left(\Sigma, \nu, \Sigma^{\prime}\right)$ is an extreme point iff for all $A \in \Sigma^{\prime}$ and $\epsilon>0$ there exists $B \in \Sigma$ with $\mu(A \triangle B)<\epsilon$.

Proof. Let $\left(S_{1}, \Sigma_{1}{ }^{\prime}\right)$ in the terminology of [4] denote the Stonian space of $\left(S, \Sigma^{\prime}\right)$ and $\tau$ the isomorphism of $\Sigma^{\prime}$ onto the field $\Sigma_{1}{ }^{\prime}$ of open and closed subsets of $S_{1} ; \Sigma_{1}{ }^{\prime \prime}$ is defined to be the $\sigma$-field generated by $\Sigma_{1}{ }^{\prime}$. Since $\tau$ induces an isomorphism $T$ of $b a(S, \Sigma)$ onto $c a\left(S_{1}, \Sigma_{1}{ }^{\prime}\right), \mu \in b a\left(\Sigma, \nu, \Sigma^{\prime}\right)$ is an extreme point iff $T(\mu) \in c a\left(\Sigma_{2}{ }^{\prime \prime}, \nu^{\prime \prime}, \Sigma_{1}{ }^{\prime \prime}\right)$ is an extreme point, where $\Sigma_{2}$ " is the $\sigma$-field which is generated by the field $\Sigma_{2}{ }^{\prime}=\tau(\Sigma)$, and $\nu^{\prime \prime} \in c a\left(S_{1}, \Sigma_{2}{ }^{\prime \prime}\right)$ is the (uniquely determined) extension of $\nu^{\prime}$ ' defined by $\nu^{\prime}(B)=\nu\left(\tau^{-1}(B)\right), B$ $\in \Sigma_{2}{ }^{\prime}$. Furthermore $\mu^{\prime}=T(\mu) \in c a\left(\Sigma_{2}{ }^{\prime \prime}, \nu^{\prime \prime}, \Sigma_{1}{ }^{\prime \prime}\right)$ is an extreme point iff for all $A_{1} \in \Sigma_{1}$ " there exists $B_{1} \in \Sigma_{2}$ " with $\mu^{\prime}\left(A_{1} \triangle B_{1}\right)=0$. Finally for all $A_{1} \in \Sigma_{1}{ }^{\prime \prime}$, resp. $B_{1} \in \Sigma_{2}{ }^{\prime \prime}$, and $\epsilon>0$, there is a $C_{1} \in \Sigma_{1}{ }^{\prime}$, resp. $D_{1} \in \Sigma_{2}{ }^{\prime \prime}$, such that $\mu^{\prime}\left(A_{1} \triangle C_{1}\right)<\epsilon$, resp. $\mu^{\prime}\left(B_{1} \triangle D_{1}\right)<\epsilon$, holds [1, p. 21]. From this Theorem 1 follows if one notices that $\tau$ is an isomorphism of $\Sigma^{\prime}$ onto $\Sigma_{1}{ }^{\prime}$ and

Received by the editors November 4, 1974.

AMS (MOS) subject classifications (1970). Primary 28A10.

Key words and phrases. Extreme points, finitely additive set functions, $\{0,1\}$-valued set functions, Baire resp. Borel measures. 
that with the help of the symmetric difference and $\mu^{\prime}$ a pseudometric with respect to $\Sigma_{1} "$ is defined.

REMARKS. 1. If one chooses for $\Sigma$ the trivial field $\{\varnothing, S\}$, one gets a result of Choquet [2, p. 245], who characierized the extreme points of the set of all $\mu \in b a(S, \Sigma)$ with $\mu \geq 0$ and $\mu(S)=1$ by the $\{0,1\}$-valued ones.

2. If $\Sigma^{\prime}$ can be generated by adjoining a system $\Gamma$ of subsets of $S$ to $\Sigma$, the approximation property of Theorem 1 is satisfied for all $A \in \Sigma$ iff it holds for all $G \in \Gamma$, because the class of all $A \in \Sigma^{\prime}$ with this approximation property is a field which contains $\Sigma$ and $\Gamma$.

3. If $\Sigma^{\prime}$ can be generated by adjoining a countable system $\Gamma$ of subsets of $S$ to $\Sigma$, the set of extreme points of $b a\left(\Sigma, \nu, \Sigma^{\prime}\right)$ is a $G_{\delta^{-}}$-set in the weak* topology of $b a\left(S, \Sigma^{\prime}\right)$ without Choquet's metrizability assumptions as the example $S=\mathbf{N}, \Sigma=\{\varnothing, S\}$ and $\Sigma^{\prime}=\wp(S)$ shows because of [4, p. 426].

ExAmples. 1. Let $\Gamma$ be equal to $\{G\}$. Then $\not$ ós and Marczewski [6] have shown, that $\mu_{i}, i=1,2$, defined by $\mu_{i}\left(B_{1} G+B_{2} G^{c}\right)=\nu^{*}\left(B_{1} G\right)$ $+\nu_{*}\left(B_{2} G^{c}\right)$ for $i=1$, resp., $\nu_{*}\left(B_{1} G\right)+\nu^{*}\left(B_{2} G^{c}\right)$ for $i=2$ and for all $B_{j}$ $\in \Sigma, j=1,2$, are elements of $b a\left(\Sigma, \nu, \Sigma^{\prime}\right)$, where $\nu^{*}$, resp. $\nu_{*}$, is the outer, resp. inner, measure of $\nu$. From Theorem 1 and the following remarks one concludes, that they are extreme points of $b a\left(\Sigma, \nu, \Sigma^{\prime}\right)$.

2 . If $S$ is a compact topological space, $\Sigma$, resp. $\Sigma^{\prime}, \sigma$-fields of Baire, resp. Borel, subsets of $S$, then a Baire (probability) measure $\nu_{0}$ can be uniquely extended to a regular Borel measure $\mu_{0}$, from which follows that $\mu_{0}$ is an extreme point of $c a\left(\Sigma, \nu_{0}, \Sigma^{\prime}\right)$ (it is not difficult to prove that $\mu_{0}$ is even an exposed point in the sense that there is a set $A_{0} \in \Sigma^{\prime}$, take, for example, the support of $\mu_{0}$, such that $\mu_{0}\left(A_{0}\right)=1>\mu\left(A_{0}\right)$ for all $\mu \in c a\left(\Sigma, \nu_{0}, \Sigma^{\prime}\right)$ with $\left.\mu \neq \mu_{0}\right)$. Hence for all $A \in \Sigma^{\prime}$ there is a $B \in \Sigma$ with $\mu_{0}(A \triangle B)=0$. This is a known result (Berberian [1, p. 221]).

Whereas in the case $c a\left(\Sigma, \nu, \Sigma^{\prime}\right)$ the set of extreme points may be empty (take, for example, for $S$ the set of real numbers, $\Sigma=\left\{B \subset S \mid B\right.$, resp. $B^{c}$, is countable $\}, \Sigma^{\prime}$ is defined to be the set of Borel subsets of $S$, and $\nu$ is defined by $\nu(B)=0$, resp. 1 , if $B$, resp. $B^{c}$, is countable), from the theorem of Krein and Milman and from $b a\left(\Sigma, \nu, \Sigma^{\prime}\right) \neq \varnothing$ follows

Corollary. Every $\nu \in b a(S, \Sigma)$ with $\nu \geq 0$ and $\nu(S)=1$ can be extended to $\mu \in b a\left(S, \Sigma^{\prime}\right)$ with $\mu \geq 0$ and $\mu(S)=1$, such that for all $A \in \Sigma$ and $\epsilon>0$ there is a $B \in \Sigma$ with $\mu(A \triangle B)<\epsilon$.

REMARK. For the extension $\mu$ of $\nu$ in the Corollary, it holds that the closures (in the topology of the set of real numbers) of the range of $\nu$, resp. of $\mu$, coincide. The existence of extensions with this property are proved by Sikorski and Tarski (see [6]). If the extension $\mu$ of $\nu$ is unique, it follows from $b a\left(\Sigma, \nu, \Sigma^{\prime}\right) \neq \varnothing$ and Example 1 that a stronger approximation property for $\mu$ holds.

THEOREM 2. $b a\left(\Sigma, \nu, \Sigma^{\prime}\right)=\{\mu\}$ holds iff for all $A \in \Sigma^{\prime}$ and $\epsilon>0$ there exists $B_{i} \in \Sigma, i=1,2$, with $B_{1} \subset A \subset B_{2}$ and $\nu\left(B_{2} \backslash B_{1}\right)<\epsilon$.

REMARKS. 1. Theorem 2 holds in the case $c a\left(\Sigma, \nu, \Sigma^{\prime}\right)=\{\mu\}$ if it is possible to extend probability measures on $\sigma$-fields $\Sigma^{\prime \prime}\left(\Sigma \subset \Sigma^{\prime \prime} \subset \Sigma^{\prime}\right)$ to $\Sigma^{\prime}$, for example if $S$ is countable (see [5]). But even in the case where $S$ is compact 
and $\Sigma$, resp. $\Sigma^{\prime}$, is the set of Baire, resp. Borel, subsets of $S$, the countably additive version of Theorem 2 is false. Choose, for example, a set $S^{\prime}$ with card $\left(S^{\prime}\right)=\aleph_{1}$ and equip $S$ with the discrete topology. If $S=S^{\prime} \cup\{\infty\}$ denotes the one point compactification of $S$, then $\Sigma$ consists of all countable subsets of $S^{\prime}$ and their complements with respect to $S$ and $\Sigma^{\prime}=\wp(S)$. Now on $\wp(S)$ exist because of a theorem of Ulam [7] only discrete probability measures, which implies that for the Dirac measure $\delta_{\infty}$ on $\Sigma^{\prime}$ it holds $c a\left(\Sigma, \delta_{\infty} \mid \Sigma, \Sigma^{\prime}\right)=\delta_{\infty}$, but does not have the approximation property of Theorem 2. This answers two questions of Berberian [1, p. 233], whether a monogenic Baire measure is always completely regular in the sense of Theorem 2 and whether $\Sigma$ is equal to $\Sigma^{\prime}$ if all Baire measures are monogenic.

2. Theorem 2 implies that $\nu \in b a(S, \Sigma), \nu \geq 0, \nu(S)=1$ can be uniquely decomposed in the following way: $\nu=a \nu_{1}+(1-a) \nu_{2}, a \in[0,1]$, where $\nu_{1}$ $\in b a(S, \Sigma), \nu_{1} \geq 0, \nu_{1}(S)=1$ can be uniquely extended to $\mu_{1} \in b a(S, \Sigma)$ with $\mu_{1} \geq 0$ and with $\nu_{2} \in b a(S, \Sigma), \nu_{2} \geq 0, \nu_{2}(S)=1$ such that $\nu_{2}$ is singular with respect to all $\nu_{1}^{\prime} \in b a(S, \Sigma)$ with this property. Furthermore $\nu_{1}$ is given by: $a \nu_{1}(B)=\inf \left\{\nu_{*}\left(A_{1}\right)+\cdots+\nu_{*}\left(A_{n}\right) \mid A_{i} \in \Sigma\right.$ pairwise disjoint, $\left.i=1, \ldots, n, \cup_{i=1}^{n} A_{i}=B\right\}$, where $\nu_{*}$ is the inner measure of $\nu$ (restricted to $\Sigma)$.

Finally a simple decomposition property will be derived, which $\nu$ must have if $b a\left(\Sigma, \nu, \Sigma^{\prime}\right)$ has at most $r$ extremal points. For this purpose let $B_{i} \in \Sigma, i$ $=1, \ldots, s$, pairwise disjoint with $\cup_{i=1}^{s} B_{i}=S$ and $\left\{\mu_{1}, \ldots, \mu_{s}\right\}, s \leq r$, the set of extreme points of $b a\left(\Sigma, \nu, \Sigma^{\prime}\right)$. Then $\mu \in b a\left(\Sigma, \nu, \Sigma^{\prime}\right)$ defined by $\mu(A)$ $=\mu_{1}\left(B_{1} A\right)+\cdots+\mu_{s}\left(B_{s} A\right)$ for all $A \in \Sigma^{\prime}$ has the approximation property in Theorem 1 which implies that there is a $t \in\{1, \ldots, s\}$ such that $\mu_{t}$ $=\mu_{i}$ on $B_{i} \Sigma$ for all $i \in\{1, \ldots, s\}$. Since this property holds for the restriction of $\nu$ to $B \Sigma$ for an arbitrary $B \in \Sigma$ one yields

THEOREM 3. If $b a\left(\Sigma, \nu, \Sigma^{\prime}\right)$ has at most $r \geq 2$ extreme points, then there exist $a_{i} \in[0,1], i=1, \ldots, s$, with $\sum_{i=1}^{s} a_{i} \leq 1$, and $\{0,1\}$-valued finitely additive set functions $\nu_{i}, i=1, \ldots, s$, on $\Sigma$, such that $\nu=\sum_{i=1}^{s} a_{i} \nu_{i}+\left(1-\sum_{i=1}^{s} a_{i}\right) \nu_{0}$ holds, where $s=\left(\begin{array}{l}r \\ 2\end{array}\right)$ and $\nu_{0} \in b a(S, \Sigma), \nu_{0} \geq 0, \nu_{0}(S)=1$, and $\nu_{0}$ has the approximation property in Theorem 2 .

Proof. Induction with respect to $r$ together with a maximal (with respect to inclusion) system $\left\{B_{i} \mid B_{i} \in \Sigma, i \in I\right.$, pairwise disjoint and for all $i \in$ $I$ the corresponding $B_{i}$ can be decomposed in at least $r$ pairwise disjoint subsets with positive measure $\nu$ \} implies Theorem 3.

REMARK. By simple examples it is seen that the number $s=\left(\begin{array}{l}r \\ 2\end{array}\right)$ is minimal in the representation.

I wish to thank the referee for valuable remarks.

\section{REFERENCES}

1. S. K. Berberian (1965), Measure and integration, Macmillan, New York. MR 32 \# 1315.

2. G. Choquet (1953/54), Theory of capacities, Ann. Inst. Fourier (Grenoble) 5, 131-295 (1955). MR 18, 295.

3. R. G. Douglas (1966), On extremal measures and subspace density. II, Proc. Amer. Math. Soc. 17, 1363-1365. MR 34 \#4888.

4. N. Dunford and J. T. Schwartz (1964), Linear operators. I: General theory, Pure and Appl. 
Math., vol. 7, Interscience, New York. MR 22 \#8302.

5. H. Hanisch, W. M. Hirsch and A. Renyi (1969), Measures in denumerable spaces, Amer. Math. Monthly 76, 494-502. MR 39 \#4352.

6. J. Lós and E. Marczewski (1949), Extensions of measure, Fund. Math. 36, 267-276. MR 11, 717.

7. S. M. Ulam (1930), Zur Masstheorie in der allgemeinen Mengenlehre, Fund. Math. 16, 141-150.

Institut für Mathematische Statistik der Universität, D-44 Münster, Roxeler Str. 64, MÜNSTER, GeRMANY 